\title{
BMJ Open A pilot randomised controlled trial of an energy management programme for adults on maintenance haemodialysis: the fatigue-HD study
}

\author{
Janine F Farragher (D) , ${ }^{1}$ Pietro Ravani, ${ }^{2}$ Braden Manns, ${ }^{3}$ Meghan Elliott (D) , ${ }^{3}$ \\ Chandra Thomas, ${ }^{2}$ Maoliosa Donald, ${ }^{3}$ Nancy Verdin, ${ }^{4}$ Brenda R Hemmelgarn ${ }^{2,4}$
}

To cite: Farragher JF, Ravani P, Manns B, et al. A pilot randomised controlled trial of an energy management programme for adults on maintenance haemodialysis: the fatigue-HD study. BMJ Open 2022;12:e051475. doi:10.1136/ bmjopen-2021-051475

\section{- Prepublication history and} additional supplemental material for this paper are available online. To view these files, please visit the journal online (http://dx.doi.org/10.1136/ bmjopen-2021-051475).

Received 10 April 2021 Accepted 20 January 2022

Check for updates

(c) Author(s) (or their employer(s)) 2022. Re-use permitted under CC BY-NC. No commercial re-use. See rights and permissions. Published by BMJ.

${ }^{1}$ Department of Occupational Science \& Occupational Therapy, University of Toronto, Toronto, Ontario, Canada

${ }^{2}$ Department of Medicine, University of Calgary, Calgary, Alberta, Canada

${ }^{3}$ Department of Community Health Sciences, University of Calgary, Calgary, Alberta, Canada

${ }^{4}$ Department of Medicine, University of Alberta, Edmonton, Alberta, Canada

Correspondence to

Dr Janine F Farragher;

janine.farragher@utoronto.ca

\section{ABSTRACT}

Background Identifying interventions to reduce fatigue and improve life participation are top research priorities of people on maintenance haemodialysis.

Objective Our primary objective was to explore the feasibility of conducting a randomised controlled trial of an energy management programme for people on maintenance haemodialysis.

Design Parallel-arm, 1:1, blinded, pilot randomised controlled trial.

Participants Participants were recruited from 6 dialysis units in Calgary, Canada. Eligible patients were on maintenance haemodialysis, clinically stable and reported disabling fatigue on the Fatigue Severity Scale items 5, 7, 8 and 9.

Randomisation Participants were randomised using a computer-generated random number sequence according to permuted blocked randomisation, stratified by dialysis unit.

Blinding Participants were blinded to treatment allocation.

Interventions Participants received an attention control (general disease self-management education) or the Personal Energy Planning (PEP) programme, a tailored, web-supported 7-9 weeks energy management programme.

Outcomes Eligibility, recruitment and attrition rates were recorded, and standardised intervention effects (Hedge's

G) were calculated for fatigue and life participation questionnaires at one1-week postintervention and 12week postintervention.

Results 159 of 253 screened patients were eligible to be approached. 42 (26\%) had fatigue, were interested and consented to participate, of whom 30 met eligibility criteria and were randomised (mean age 62.4 years $( \pm 14.7)$, $60 \%$ male). 22 enrolled participants $(73 \%)$ completed all study procedures. Medium-sized intervention effects were observed on the Canadian Occupational Performance Measure (COPM)-Performance Scale, Global Life Participation Scale and Global Life Participation Satisfaction Scale at 1-week postintervention follow-up, compared with control. At 12-week follow-up, large and very large intervention effects were observed on the COPM-Performance Scale and COPM-Satisfaction Scale, respectively.
Strengths and limitations of this study

- We referenced the Standard Protocol Items: Recommendations for Interventional Trials guidelines for a pilot randomised controlled trial throughout the development and writing of the trial protocol, and used a standardised intervention training protocol to maximise treatment fidelity across programme administrators.

- We used randomisation, participant blinding and an active control group to control for bias.

- Required proficiency in English means results might not be generalisable to non-English-speaking populations.

- Unequal attrition rates between the intervention and control groups limit the conclusions that can be drawn about programme efficacy from this pilot study, underscoring the need for further research to confirm these preliminary findings.

Conclusion It is feasible to enrol and follow patients on haemodialysis in a randomised controlled trial of an energy management intervention. As the intervention was associated with improved life participation on some measures, a larger trial is justified.

\section{INTRODUCTION}

Kidney failure is associated with a variety of symptoms, including pain, nausea and insomnia, which can affect quality of life. $^{12}$ One of the most challenging symptoms, chronic fatigue, ${ }^{3}$ is experienced by an estimated $70 \%$ of the population with kidney failure on maintenance haemodialysis. ${ }^{1}$ Fatigue can negatively affect various aspects of well-being in people with kidney failure, including mood, motivation and quality of life. ${ }^{45}$ However, its negative impact on their ability to participate in valued life activities (ie, life participation) has been identified as their top priority for research and intervention. ${ }^{5}$ People on haemodialysis have described limitations in their ability to 
perform valued activities, such as work, socialising and household management, because of fatigue. ${ }^{4-6}$ They have indicated that the ability to participate in life activities should be a key indicator of treatment effectiveness. ${ }^{5}$ However, evidence-based treatments to reduce fatigue or mitigate its impact on life participation are limited for this population. There are a complex and poorly understood range of factors that contribute to kidney disease fatigue, including anaemia, chronic inflammation, malnutrition and depression, ${ }^{7}$ which limits efficacious treatments. Erythropoeitin-stimulating agents (ESAs) and exercise training are currently the primary evidence-based approaches for treating fatigue in this population ${ }^{8}$; however, ESAs are already used in a large proportion of patients, and exercise training is challenging to promote in this patient group because of several factors including inadequate staff expertise, competing patient symptoms and low motivation among patients to participate in exercise. ${ }^{111}$ There is therefore a need to explore alternative approaches that can help people with kidney disease fatigue participate in valued life activities.

Energy management education (EME) aims to improve life participation in people with fatigue by providing strategies to conserve or reallocate energy during routine daily activities. ${ }^{12}$ The theory underlying energy management is that life participation can be improved in people with chronic fatigue by minimising the exertional fatigue associated with performing daily activities ${ }^{12}{ }^{13}$; this exertional fatigue could either be a casual or exacerbating factor in the underlying fatigue and disability experienced in many chronic diseases, including kidney disease. Energy management strategies can include prioritising, changing body postures, organising the home environment or using assistive tools (eg, mobility aids, long-handled reachers). ${ }^{14}$ The Personal Energy Planning (PEP) programme is an energy management programme designed to improve life participation in people with kidney failure, by helping patients identify energy management strategies that can facilitate their individual life participation goals. ${ }^{15}$ Proofof-concept evidence has suggested the PEP programme might be associated with improvements in life participation and/or fatigue in dialysis patients, ${ }^{16}$ justifying the need for further evaluation with a randomised controlled trial (RCT). However, recruitment for RCTs can be challenging in people with kidney failure, ${ }^{17}$ in part due to a reluctance among dialysis patients to participate in research studies that require extra study-related activities or visits. ${ }^{18}$ Furthermore, the acceptability of, and interest in, the energy management approach has never been explored in people on maintenance haemodialysis.

We designed an RCT of the 'PEP' energy management $\operatorname{program}^{19}$ that attempts to minimise study burden by using simple communication materials (eg, a brochurestyle consent form); brief questionnaires; concise intervention sessions and a flexibility around missed or delayed treatment sessions. However, the feasibility of recruiting and retaining participants for a trial of an energy management programme remains unknown. More information is also needed about how the 'PEP' programme impacts various facets of life participation and fatigue, to inform the choice of a primary outcome measure and aid power calculations for an RCT.

The primary objective of our pilot trial was to estimate the proportion of patients on maintenance haemodialysis that met eligibility criteria, agreed to participate and completed all study procedures for an RCT of the 'PEP' EME programme. Our secondary objective was to estimate the effects of the programme on various facets of fatigue and life participation, to ensure a trial will be adequately powered and will use the most appropriate primary outcome measure.

\section{METHODS \\ Study design}

We conducted a multisite, parallel-group, 1:1, pilot RCT ${ }^{19}$ (www.clinicaltrials.gov; NCT03825770). We randomised 30 participants on maintenance haemodialysis to undergo the PEP energy management programme, or an active control (general self-management support).

\section{Participants}

We recruited participants on maintenance haemodialysis therapy at six haemodialysis units from 1 February 2019 to 27 August 2019. We sought patients aged $\geq 18$ years who were undergoing haemodialysis for $\geq 3$ months at time of recruitment; were clinically and cognitively stable (able to provide informed consent) and scored an average of $\geq 4$ on items 5, 7, 8 and 9 from the Fatigue Severity Scale ${ }^{20}$ (ie, items that assess the impact of fatigue on life participation). We excluded patients if they had a plan in place to discontinue in-centre haemodialysis within 6 months of recruitment; if they had inadequate written and verbal English comprehension for study activities; if they resided in a long-term care facility or if they had a visual impairment that would preclude them from engaging with study materials. Original exclusion criteria also included a score of $>3$ on the Patient Health Questionnaire-2 depression tool; however, this was subsequently removed due to interest from patients in participating in the study, and a lack of conclusive evidence that depression would impede study participation or outcomes. Instead, we measured and monitored depression at baseline in all enrolled participants.

We approached patients identified by clinical staff as being clinically and cognitively stable and Englishspeaking, to assess their interest in the study. We then enrolled and randomised eligible and consenting patients into the study.

\section{Randomisation and blinding}

We allocated participants equally (1:1) to intervention or control, using a computer-generated random number sequence. We used permuted blocked randomisation, with block sizes of 2-6, stratified by dialysis unit. We concealed allocation by having a research manager not otherwise 
Table 1 Description of the Personal Energy Planning programme

\begin{tabular}{|c|c|}
\hline $\begin{array}{l}\text { Programme } \\
\text { section }\end{array}$ & Description \\
\hline $\begin{array}{l}\text { Part 1: } \\
\text { computer } \\
\text { modules }\end{array}$ & $\begin{array}{l}\text { Participants complete three computer modules over three sessions ( 20-30 min each) that explain the } \\
\text { basic principles of energy management. } \\
\text { Modules are completed on laptops during haemodialysis sessions, with support for module completion } \\
\text { provided by study coordinators. }\end{array}$ \\
\hline $\begin{array}{l}\text { Part 2: } \\
\text { individualised } \\
\text { problem- } \\
\text { solving }\end{array}$ & $\begin{array}{l}\text { Participants work 1:1 with a trained administrator over four to six sessions ( } 30 \text { min each) to develop } \\
\text { energy management strategies for three life participation goals. } \\
\text { Energy management strategies are developed using a metacognitive problem-solving process called 'Goal- } \\
\text { Plan-Do-Check': } \\
\text { 1. Set a life participation goal; } \\
\text { 2. Analyse current energy expenditure patterns to come up with a plan to conserve energy for the goal; } \\
\text { 3. Do the plan; } \\
\text { 4. Check to see if it worked, and what aspects of the plan should be revised. } \\
\text { This process continues until an effective plan is found for each goal, or the programme maximum of } 9 \\
\text { weekly treatment sessions is reached. } \\
\text { Study coordinators use guided discovery teaching to encourage patient independence in working through } \\
\text { the Goal-Plan-Do-Check process. }\end{array}$ \\
\hline
\end{tabular}

involved with the study, provide treatment allocation to study coordinators over the phone. Study participants were blinded to their treatment status (intervention or active control). It was not feasible to blind study coordinators, given the extensive training they received to learn to administer the intervention compared with the control.

\section{Intervention: the 'PEP' programme}

Participants randomised to the treatment arm completed the tailored, 7-9 weeks PEP programme ${ }^{14}{ }^{18}$ (see table 1 for further information). The PEP programme is a twopart intervention that teaches participants how to use energy management strategies (eg, simplifying tasks, pacing, using assistive devices, organising home environments) to improve participation in three self-selected life activities. In the first part of the intervention, participants complete three web modules that define and explain the energy management approach, and describe a structured strategy for problem-solving around fatigue. In the second part of the intervention, participants work 1:1 with a study coordinator during four to six sessions to apply the principles and strategies from part 1, and problemsolve around their fatigue problems to accomplish three life participation goals (eg, cook dinner twice per week; garden in the backyard more frequently). The number of individual sessions during this part was determined by individual patient needs and progress.

Study coordinators received in-person training in the treatment and control protocols from a trained occupational therapist prior to administering the intervention. Training consisted of three in-person training sessions, led by an occupational therapist (JFF), on the core facilitation skills of the problem-solving method used in PEP (clientchosen goals, guided discovery, global problem-solving strategy, dynamic performance analysis and energy management strategies). They were also provided with a written guidebook, including suggested scripts to introduce key concepts; example dialogues between coach and patients and analysis questions and suggested energy management suggestions for various possible life participation goals. Study coordinators monitored and encouraged participant adherence to the treatment protocol during weekly visits. Missed or incomplete intervention sessions were documented and addressed as outlined in the study protocol. ${ }^{19}$

\section{Control: general information about kidney disease}

Participants randomised to the control arm reviewed general information about kidney disease management (eg, blood pressure management; diet; communicating with healthcare team) from the Kidney School online learning modules during six to eight 1:1 sessions with a trained study coordinator. Sessions took place while participants were undergoing haemodialysis.

\section{Data collection}

Trained study coordinators collected baseline demographic and clinical data on participants at the time of the first study visit, through chart review and/or participant interview. The study coordinators tracked the number of screened patients who met study eligibility criteria, consented to participate and completed all study procedures (intervention and assessment sessions), using study logs. The study coordinators administered a series of selfreported questionnaires measuring life participation and fatigue (see table 2 for list of measures and details), at three timepoints:

1. Preintervention baseline;

2. One week after the PEP programme was completed;

3. Twelve weeks after the PEP programme was completed.

Participants completed study questionnaires during their haemodialysis sessions.

\section{Statistical analyses}

We calculated the proportion of patients on haemodialysis meeting each of the feasibility end points (study 


\section{Table 2 Life participation and fatigue outcome measures}

\begin{tabular}{|c|c|c|}
\hline Outcome & Measure & Description \\
\hline \multirow[t]{3}{*}{ Life participation } & $\begin{array}{l}\text { Canadian Occupational } \\
\text { Performance Measure- } \\
\text { Performance Subscale }^{26}\end{array}$ & $\begin{array}{l}\text { Asks individuals to rate, on a 10-point Likert scale, his/her performance in each } \\
\text { of three self-selected priority activities of everyday living. Higher scores out of } 10 \\
\text { indicate better performance. The COPM has been found to be a valid, reliable, } \\
\text { clinically useful and responsive measure of occupational performance in multiple } \\
\text { chronic disease populations. }{ }^{27}\end{array}$ \\
\hline & $\begin{array}{l}\text { COPM-Satisfaction } \\
\text { Subscale }^{26}\end{array}$ & $\begin{array}{l}\text { Asks individuals to rate, on a 10-point Likert scale, their satisfaction with their } \\
\text { performance in three self-selected priority activities of everyday living. Higher scores } \\
\text { out of } 10 \text { indicate better satisfaction with performance. }\end{array}$ \\
\hline & $\begin{array}{l}\text { Reintegration to Normal } \\
\text { Living Index (RNLI) }^{28}\end{array}$ & $\begin{array}{l}\text { Assesses the degree to which individuals who have experienced traumatic or } \\
\text { incapacitating illness achieve reintegration into normal activities, using } 11 \text { declarative } \\
\text { statements each accompanied by a } 10 \text {-point visual analogue scale. Scores are then } \\
\text { added to produce an overall score out of } 110 \text {, with higher scores indicating better } \\
\text { reintegration to normal living. The RNLI has strong validity and reliability in multiple } \\
\text { chronic disease populations. }\end{array}$ \\
\hline \multirow[t]{2}{*}{ Fatigue } & $\begin{array}{l}\text { Fatigue Severity Scale } \\
(\text { FSS })^{20}\end{array}$ & $\begin{array}{l}\text { Includes } 9 \text { items that ask individuals to rate, on a Likert scale from } 1 \text { to } 7 \text {, the severity } \\
\text { of their fatigue and its impact on their life during the past week. Scores are then } \\
\text { summed and averaged to create a total score out of } 7 \text {, with higher scores indicating } \\
\text { worse fatigue. The FSS is a valid, reliable and responsive measure }{ }^{30} \text { that has been } \\
\text { used in the dialysis population. }\end{array}$ \\
\hline & $\begin{array}{l}\text { Modified Fatigue Impact } \\
\text { Scale (MFIS) }\end{array}$ & $\begin{array}{l}\text { A } 21 \text {-item Likert-based scale that assesses the effects of fatigue on physical, } \\
\text { cognitive and psychosocial functioning. Scores are summed to produce an overall } \\
\text { score out of } 84 \text {, with higher scores indicating worse fatigue impact. The MFIS is } \\
\text { frequently used as an outcome measure in energy management studies. }\end{array}$ \\
\hline
\end{tabular}

*Measure was finalised and added after trial registration, on consultation with the measure developers.

eligibility, enrolment and completion), with accompanying $95 \%$ CIs. We reported participant demographic and clinical data as means and SD for continuous parametric data; medians and IQRs for continuous non-parametric data and frequencies and percentages for categorical data. We then calculated raw and standardised treatment effect sizes for each life participation and fatigue outcome measure, at both the 1-week postintervention and 12-week postintervention timepoints. We used the Hedge's G statistic to calculate standardised effect sizes, and categorised effect size estimates as very small $(0.01-$ $0.20)$, small $(0.2-0.49)$, medium (0.5-0.79), large (0.81.19) or very large $(>1.20) .{ }^{21}$ Missing follow-up data were addressed using pairwise deletion.

Post hoc sensitivity analyses were performed that assumed best-case scenario for missing data (ie, the median intervention effect was imputed for missing intervention values, and the median control effect was imputed for missing control values), and worst-case scenario (ie, the median intervention effect was imputed for missing control values, and the median control effect was imputed for missing intervention values).

\section{Sample size}

We originally chose a sample size of 40 patients for the pilot trial. This was based on recommendations for optimal pilot study sample sizes,${ }^{22}$ an expected participant pool of 425 patients and our anticipated eligibility and recruitment rates. The target sample size was subsequently reduced to 30 due to an inability of our study team to follow patients on evening dialysis shifts, which reduced our potential participant pool from 425 to 253 patients.

\section{Patient and public involvement}

The study intervention was developed based on results of patient engagement research which suggested a need to further investigate fatigue in kidney disease. Two patients were involved in the development of the intervention through a series of individual interviews. Two patients 
were consulted about the acceptability of the active control used in this study. A patient partner reviewed the manuscript and provided feedback about the discussion and interpretation of results. Patient involvement resulted in refinement and improvement of both the intervention and control conditions, to enhance their acceptability to patients. Our patient partner provided valuable insights about important qualitative information to collect from patients, which was subsequently incorporated into a substudy involving a follow-up interview with study participants.
RESULTS

\section{Feasibility}

We screened all patients $(n=253)$ undergoing daytime maintenance haemodialysis at six dialysis centres between February and August 2019 for preliminary eligibility (ie, no language barrier, clinically and cognitively stable) (figure 1). All 159 patients who met preliminary criteria for the study $(63 \%$ (95\% CI $57 \%$ to $69 \%)$ ) were approached. Forty-two patients (26\% (95\% CI 20\% to $34 \%)$ ) reported fatigue, were interested in participating and provided consent. Of those, 30 patients $(71 \%$ (95\%

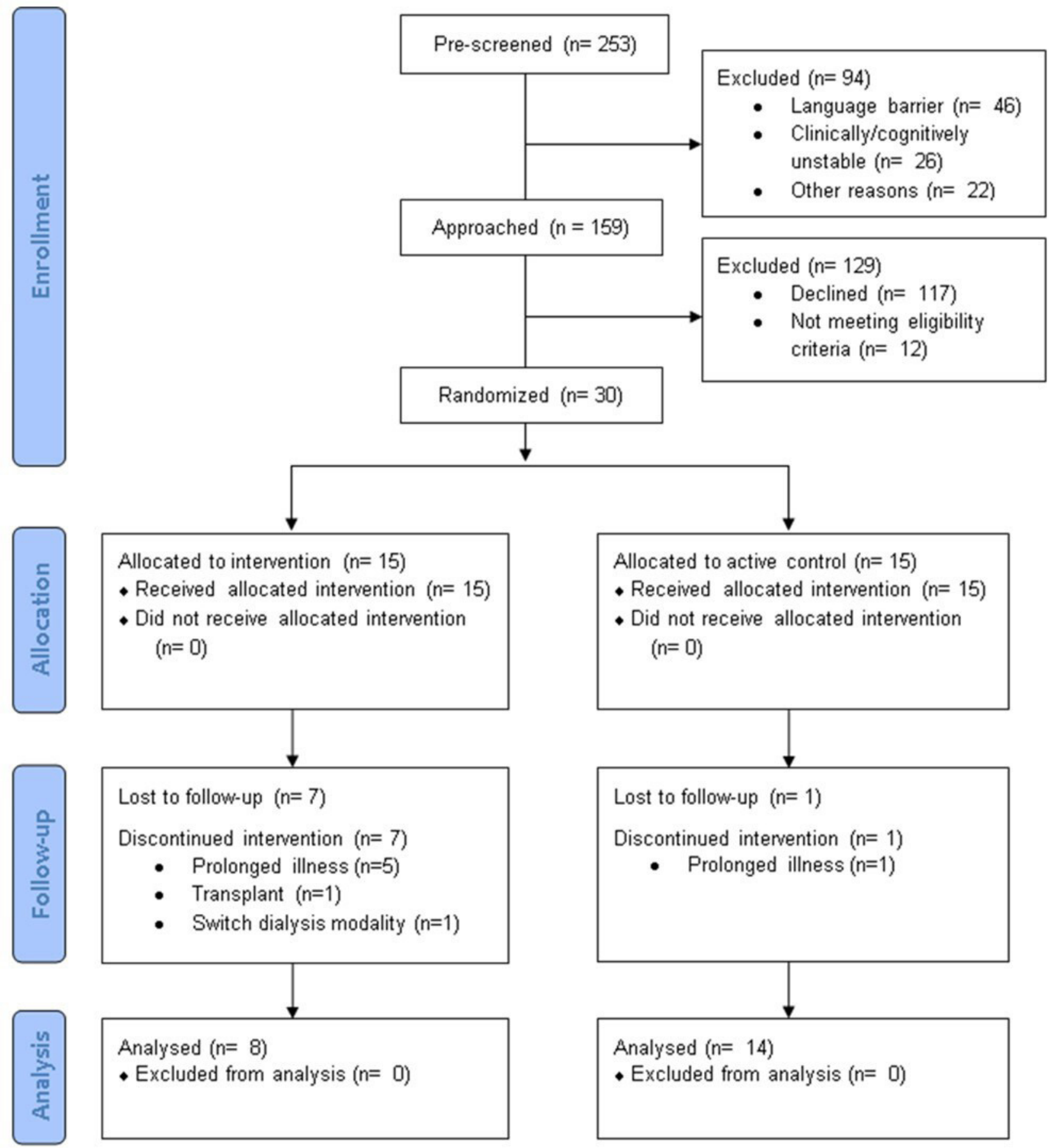

Figure 1 Consolidated Standards of Reporting Trials participant flow diagram. 
CI $55 \%$ to $84 \%$ )) met full study eligibility criteria and were enrolled and randomised. In total, 30 of 159 clinically stable and English-speaking patients (19\%, 95\% CI $13 \%$ to $25 \%$ ) were enrolled in the study.

Twenty-two of 30 enrolled patients $(73 \%$ (95\% CI $54 \%$ to $88 \%$ )) completed all study procedures: 8 in the intervention group and 14 in the control group. Reasons for study discontinuation in the intervention group included: hospitalisation or illness due to nephrectomy $(\mathrm{n}=1)$, hypoxia $(\mathrm{n}=1)$, neurological symptoms $(\mathrm{n}=1)$ or unknown reason $(n=1)$; low blood pressure during dialysis $(\mathrm{n}=1)$; switching dialysis modalities $(\mathrm{n}=1)$ and kidney transplantation $(n=1)$. The reason for discontinuation in the control group was hospitalisation due to unknown reason $(n=1)$.

\section{Participant characteristics}

Baseline characteristics of participants are described in table 3. The mean age of participants was 62.4 years $(\mathrm{SD}=14.7), 60 \%$ were male and $50 \%$ had diabetes. Participants had been on dialysis for a median of 3.6 years (IQR $1.8,7.3$ ), and $77 \%$ were living independently at baseline. Thirty per cent of participants screened positively for cognitive impairment, and $40 \%$ screened positively for depression. Participant characteristics were similar across treatment and control groups (table 3 ).

Table 3 Baseline characteristics of participants

\begin{tabular}{|c|c|c|c|}
\hline & $\begin{array}{l}\text { All participants } \\
(n=30)\end{array}$ & $\begin{array}{l}\text { Control } \\
(n=15)\end{array}$ & $\begin{array}{l}\text { Intervention } \\
(n=15)\end{array}$ \\
\hline Age (years) (mean, SD) & $62.4(14.7)$ & $64.8(14.4)$ & $60.0(15.1)$ \\
\hline Male & $18(60)$ & $10(67)$ & $8(53)$ \\
\hline \multicolumn{4}{|l|}{ Residence } \\
\hline Independent living & $27(90)$ & $14(93)$ & $13(86)$ \\
\hline Retirement/Supported living & $3(10)$ & 1 & 2 \\
\hline Lives alone & $20(67)$ & $6(40)$ & $4(27)$ \\
\hline Married & $17(57)$ & $10(67)$ & $7(46)$ \\
\hline Employed & $4(27)$ & $0(0)$ & $4(27)$ \\
\hline \multicolumn{4}{|l|}{ Education } \\
\hline No high school diploma & $3(10)$ & $2(13)$ & $1(7)$ \\
\hline High school diploma & $12(40)$ & $6(40)$ & $6(40)$ \\
\hline College/Trade school & $10(33)$ & $5(33)$ & $5(33)$ \\
\hline University degree & $4(13)$ & $2(13)$ & $2(13)$ \\
\hline Graduate/Professional degree & $1(3)$ & $0(0)$ & $1(7)$ \\
\hline Uses computer/tablet/phone & $27(3)$ & $14(93)$ & $13(86)$ \\
\hline Dialysis vintage (years) (median, IQR) & $3.6(1.8,7.3)$ & $2.6(1.7,6.0)$ & $4.0(1.7,9.5)$ \\
\hline \multicolumn{4}{|l|}{ Comorbidities } \\
\hline Diabetes & $15(50)$ & $9(60)$ & $6(40)$ \\
\hline Depression & $9(30)$ & $3(20)$ & $6(40)$ \\
\hline Coronary artery disease & $10(33)$ & $6(40)$ & $4(27)$ \\
\hline Congestive heart failure & $8(27)$ & $3(20)$ & $5(33)$ \\
\hline Cerebrovascular disease & $3(10)$ & $3(12)$ & $0(0)$ \\
\hline Alzheimer's disease & $1(3)$ & $0(0)$ & $1(7)$ \\
\hline Multiple sclerosis & $1(3)$ & $0(0)$ & $1(7)$ \\
\hline Chronic obstructive pulmonary disease & $1(3)$ & $1(7)$ & $0(0)$ \\
\hline Cancer & $7(23)$ & $5(33)$ & $2(13)$ \\
\hline Baseline serum haemoglobin (g/L) (mean, SD) & $101.6(18.7)$ & $107.7(8.7)$ & $95.0(23.3)$ \\
\hline Baseline serum albumin (g/L) (mean, SD) & $35.0(10.8)$ & $33.0(3.9)$ & $37.2(15.0)$ \\
\hline Activities of daily living dependence & $7(23)$ & $2(13)$ & $5(33)$ \\
\hline MiniCog impaired & $9(30)$ & $4(27)$ & $5(33)$ \\
\hline Personal Health Questionnaire-2 impaired & $12(40)$ & $5(33)$ & $7(47)$ \\
\hline
\end{tabular}

${ }^{\star}$ Data are expressed as $\mathrm{n}(\%)$ unless otherwise specified. 


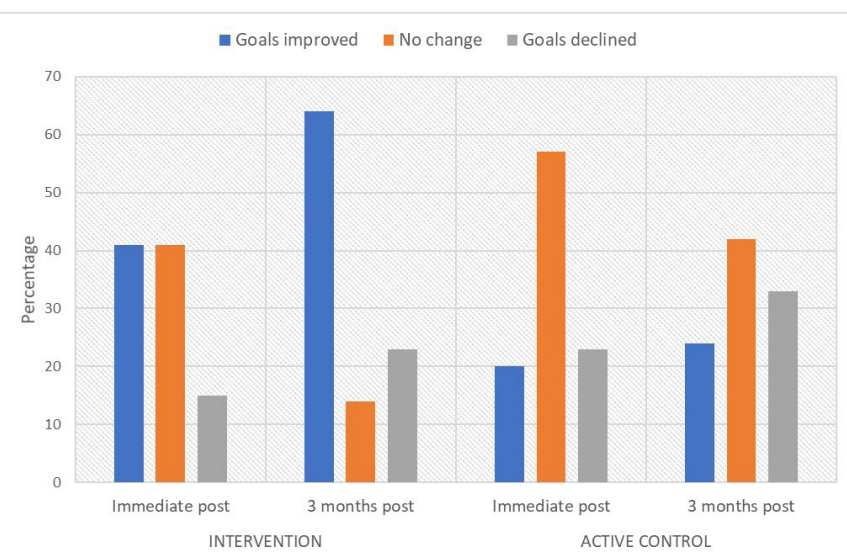

Figure 2 Proportion of patients achieving life participation goals in intervention versus control. 'Improved' means increase of $\geq 2$ points (established MCID) on COPMPerformance Subscale; 'no change' means no clinically significant change; 'declined' means decrease of $\geq 2$ points on COPM-Performance Subscale. COPM, Canadian Occupational Performance Measure; MCID, minimal clinically important difference.

\section{Effect size estimates}

We observed a large standardised intervention effect at the 1-week postintervention follow-up assessment on the COPM-Performance Scale (Hedge's G=0.62; moderate effect), compared with control. At 1-week postintervention, participants in the intervention group $(\mathrm{n}=10)$ reported a clinically meaningful improvement ( $\geq 2$ points) in $40 \%$ of their life participation goals according to the COPM-Performance Scale, compared with $21 \%$ in the active control group ( $\mathrm{n}=14$ ) (figure 2). We also observed moderate intervention effects on the Fatigue Management Questionnaire's Global Life Participation Scale (Hedge's G=0.50), Global Life Participation Satisfaction Scale (Hedge's $\mathrm{G}=0.50$ ) and Self-Efficacy Scale (Hedge's $\mathrm{G}=0.50$ ). The remainder of fatigue and life participation measures detected either small intervention effects, or no effects, at 1-week postintervention follow-up compared with control (table 4 ).

At 12 weeks postintervention, we observed large and very large effects on the COPM-Performance Scale (Hedge's G=0.90) and COPM-Satisfaction Scale (Hedge's $\mathrm{G}=1.36)$ in the intervention group $(\mathrm{n}=8)$, respectively, compared with control $(\mathrm{n}=14)$ (table 4$)$. Participants in the intervention group reported a clinically meaningful improvement ( $\geq 2$ points) in $64 \%$ of their life participation goals according to the COPMPerformance Scale at the 12-week postintervention timepoint, compared with $24 \%$ in the active control group (figure 2). We found minimal to no effects associated with the intervention on the remainder of fatigue or life participation measures at the 12-week postintervention follow-up, compared with control. Results of the sensitivity analysis, assuming best-case and worst-case scenarios for missing data, are included in Appendix 1 (online supplemental file 1 ).

\section{DISCUSSION}

In this pilot study, we assessed the feasibility of recruiting and retaining patients on maintenance haemodialysis with fatigue for an RCT of an energy management programme, and the potential impact of such a programme. Although previous proof-of-principle evidence ${ }^{16}$ suggested an RCT was warranted, the proportion of participants who would commit to completing study activities (eg, intervention sessions, outcome questionnaires) for a trial was unknown. Furthermore, the impact of the 'PEP' programme on various facets of life participation and fatigue compared with a control group remained unclear. We were able to recruit $\sim 25 \%$ of clinically stable and English-speaking haemodialysis patients into this pilot RCT, and retain $70 \%$ of enrolled participants for the duration of the trial, which met our pretrial expectations for study participation. ${ }^{19}$ Although fatigue did not appear to be affected by the PEP programme, the programme was associated with medium-sized to large-sized effect on personalised life participation at both short-term and medium-term follow-up, compared with an attention control condition. Collectively, these results suggest that an RCT of the PEP programme would be feasible, and is warranted.

Our recruitment and retainment results suggest that, despite the added responsibilities of filling out study questionnaires and completing the intervention or control programme, the study was acceptable to a substantial proportion of our target population. We note that although only $25 \%$ of stable and English-speaking haemodialysis patients consented to participate, only $50 \%-70 \%$ of them likely had fatigue, based on existing estimates of fatigue prevalence ${ }^{1}$; thus, we estimate that approximately half of eligible patients with fatigue in fact agreed to the study. This suggests that study burden was not an insurmountable barrier to recruitment. Although the dropout rate was higher in the intervention arm than the control ( $43 \%$ vs $13 \%$ ), our documented reasons for study withdrawals were unrelated to the intervention, and were rather due to the general medical complexity of this patient population. We therefore assume that with a larger sample of patients, the attrition rate would balance between the two groups. Our overall attrition rate of $30 \%$ is not unexpected for the dialysis population over the course of a 5-month study, given that they typically experience high rates of acute medical events and hospitalisations. ${ }^{23}{ }^{24}$ We attribute the general acceptability of the intervention to the use of study materials that were user-friendly (eg, a brochure-style consent form); brief questionnaires to assess target outcomes and a flexible protocol for missed treatment sessions. Acceptability could be further increased in a full-scale trial by reducing the number of questionnaires used to assess life participation and fatigue, particularly now that the pilot trial has provided clarity about the best measures for assessing these outcomes.

The finding that the PEP programme was associated with improvements in life participation, compared with control, is important because this outcome directly aligns 


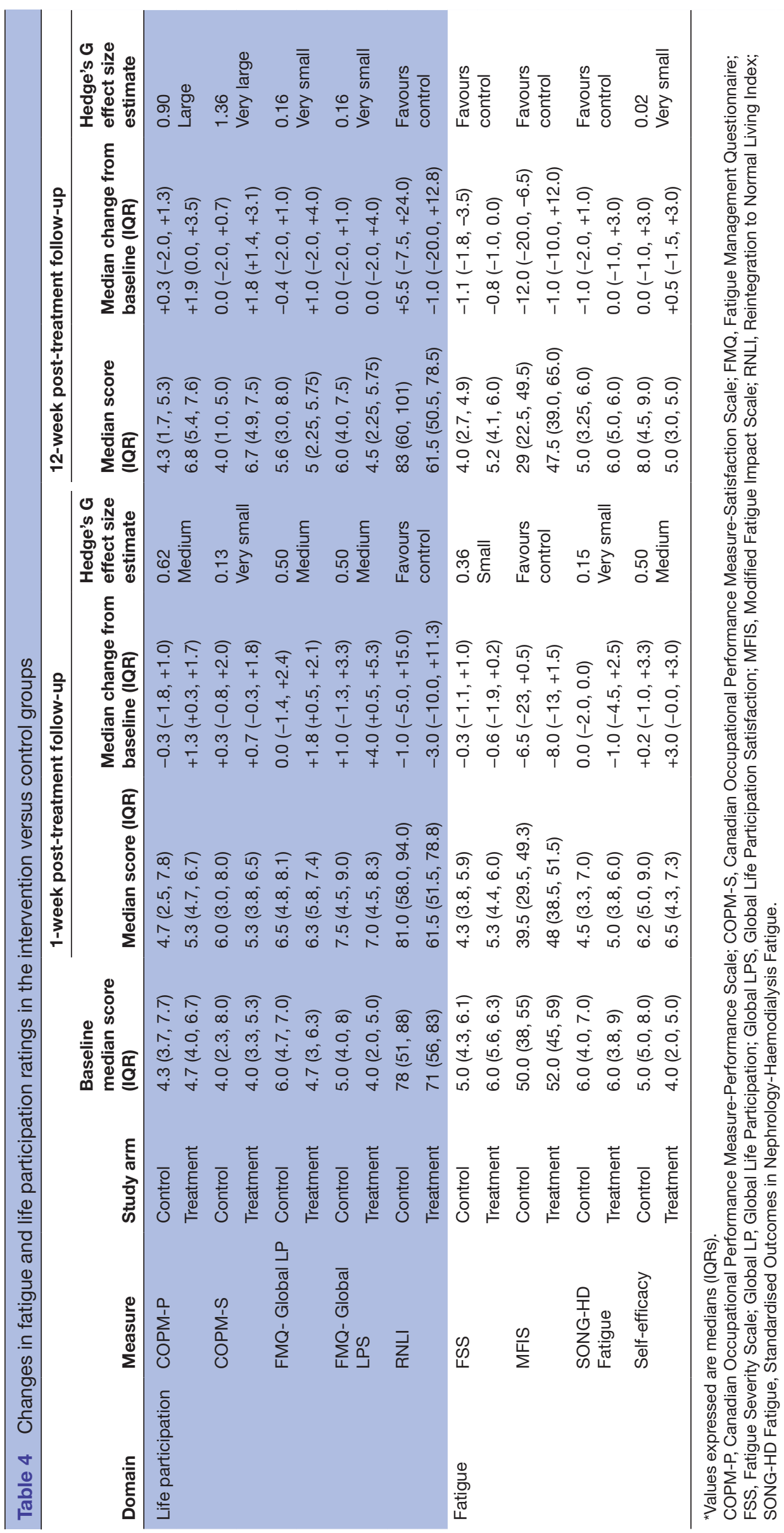


with patient priorities. ${ }^{35}$ Patients on haemodialysis with fatigue view life participation as 'the fundamental goal of treatment, because it symbolises some indicator of being able to live a life without being confined by the disease'. Although fatigue was not directly impacted by the intervention, our results suggest that energy management strategies developed during the intervention might have helped participants to accomplish their day-to-day goals more effectively by working around fatigue. In addition, the fatigue measures used in this study do not directly assess exertional fatigue (the type of fatigue targeted by the PEP programme); as such, participants might have been reporting that their underlying 'baseline' level of fatigue had not changed in response to the programme, but still might have been experiencing a reduction in exertional fatigue during valued activities. The improvement in personalised life participation we observed in this study is, nonetheless, significant and relatively unique within the energy management literature. ${ }^{25}$ Our intervention incorporated a number of novel features to more directly target life participation, compared with other energy management interventions, in accordance with the priorities of haemodialysis patients. For example, we used personalised goal-setting to ensure interventions were tailored to specific patients' needs, and a problemsolving training approach to facilitate patient independence at solving their own life participation challenges. Our findings support the potential efficacy of these features, although it is important to note the potential impact of unequal attrition between the intervention and control groups on our pilot results. This further emphasises the need for a full-scale trial to more conclusively establish programme effectiveness.

With respect to outcome measures, we found that the Canadian Occupational Performance Measure ${ }^{26}$ detected the strongest intervention effects compared with other life participation and fatigue measures. The validity and reliability of the life participation measures used have not been established in the chronic kidney disease population; as such, measures such as the Reintegration to Normal Living Index or Fatigue Management Questionnaire might not have detected intervention effects because, for example, they did not capture relevant areas or aspects of life participation among this population; were not worded in an understandable way or were not responsive enough to capture changes in the outcomes, among other potential explanations. The COPM is also the only measure we used that assessed life participation in patient-chosen activities, rather than a generic set of life activities and/or areas which might not have been relevant to the study participants. This also might explain the enhanced performance of the COPM at detecting change associated with the intervention, compared with the other life participation measures. Although the COPM has similarly not been formally validated in people with kidney failure, it has strong validity, reliability and responsiveness data from multiple other clinical populations and age groups, ${ }^{27}$ and uniquely aligns with preferences of people with kidney disease for a measure of life participation that is individualised. ${ }^{5}$ Collectively, these findings suggest the COPM is the best choice for a primary outcome for an RCT of the 'PEP' programme. Estimates based on our pilot results suggest data on 36 participants would be needed to detect a clinically meaningful change of $>2$ points on the COPM-Performance Scale in an RCT, with significance set at $80 \%$ power and $\mathrm{p}=0.05$. Based on our rates of screened-to-enrolled patients, the participant screening pool would need to include 415 patients on haemodialysis to achieve this sample size.

\section{Study limitations}

We excluded non-English-speaking patients from the study, limiting its generalisability to non-English-speaking people with kidney failure. Positive findings about the PEP programme might, however, justify developing programme materials in the future that are accessible to a wider range of people with kidney disease. We were also unable to blind study coordinators to participants' treatment allocation, which might have unduly affected their approach to treatment. The infeasibility of blinding is a well-recognised limitation of trials that study psychosocial or behavioural interventions, because of the challenges of identifying and implementing an appropriate control. Finally, unequal attrition rates between the intervention and control groups limit the conclusions that can be drawn about programme efficacy from this pilot study, and underscore the need for further research using a larger sample of patients to confirm our preliminary results.

\section{CONCLUSIONS}

The PEP energy management programme appears to be acceptable to patients, and might lead to improvements in life participation. Further investigation in an adequately powered RCT is warranted.

Twitter Janine F Farragher @janinef

Contributors JFF led the design, coordination, analysis and authorship of the study and manuscript. JFF is also acting as the guarantor. BRH provided advice and mentorship on all aspects of the study. CT, BM and MD helped with the development of the participant identification plan, and provided advice on other key study issues. PR and ME contributed feedback on trial design. All authors assisted with the interpretation and presentation of results for publication. NV provided review and perspective from a patient's point of view.

Funding JFF was supported by the Canadian Institutes of Health Research (CIHR) Fellowship Program, and the Kidney Research Scientist Core Education and National Training (KRESCENT) programme. Award/Grant numbers are not applicable.

Competing interests None declared.

Patient consent for publication Not applicable.

Ethics approval This pilot trial adhered to the principles of the Declaration of Helsinki and was approved by the Conjoined Health Research Ethics Board at the University of Calgary (\#18-1657). Participants gave informed consent to participate in the study before taking part.

Provenance and peer review Not commissioned; externally peer reviewed.

Data availability statement Data from the study can be made available on reasonable request to the corresponding author. 
Supplemental material This content has been supplied by the author(s). It has not been vetted by BMJ Publishing Group Limited (BMJ) and may not have been peer-reviewed. Any opinions or recommendations discussed are solely those of the author(s) and are not endorsed by BMJ. BMJ disclaims all liability and responsibility arising from any reliance placed on the content. Where the content includes any translated material, BMJ does not warrant the accuracy and reliability of the translations (including but not limited to local regulations, clinical guidelines, terminology, drug names and drug dosages), and is not responsible for any error and/or omissions arising from translation and adaptation or otherwise.

Open access This is an open access article distributed in accordance with the Creative Commons Attribution Non Commercial (CC BY-NC 4.0) license, which permits others to distribute, remix, adapt, build upon this work non-commercially, and license their derivative works on different terms, provided the original work is properly cited, appropriate credit is given, any changes made indicated, and the use is non-commercial. See: http://creativecommons.org/licenses/by-nc/4.0/.

\section{ORCID iDs}

Janine F Farragher http://orcid.org/0000-0002-3930-2191

Meghan Elliott http://orcid.org/0000-0002-5434-2917

\section{REFERENCES}

1 Murtagh FEM, Addington-Hall J, Higginson IJ. The prevalence of symptoms in end-stage renal disease: a systematic review. Adv Chronic Kidney Dis 2007;14:82-99.

2 Davison SN, Jhangri GS. Impact of pain and symptom burden on the health-related quality of life of hemodialysis patients. J Pain Symptom Manage 2010;39:477-85.

3 Manns B, Hemmelgarn B, Lillie E, et al. Setting research priorities for patients on or nearing dialysis. Clin J Am Soc Nephrol 2014;9:1813-21.

4 Jacobson J, Ju A, Baumgart A, et al. Patient perspectives on the meaning and impact of fatigue in hemodialysis: a systematic review and thematic analysis of qualitative studies. Am J Kidney Dis 2019;74:179-92.

5 Ju A, Unruh M, Davison S. Establishing a core outcome measure for fatigue in patients on hemodialysis: a standardized outcomes in Nephrology-Hemodialysis (SONG-HD) consensus workshop report. Am J Kidney Dis 2018.

6 Heiwe S, Dahlgren MA. Living with chronic renal failure: coping with physical activities of daily living. Adv Physiother 2004;6:147-57.

7 Artom M, Moss-Morris R, Caskey F, et al. Fatigue in advanced kidney disease. Kidney Int 2014;86:497-505.

8 Johansen KL, Finkelstein FO, Revicki DA, et al. Systematic review of the impact of erythropoiesis-stimulating agents on fatigue in dialysis patients. Nephrol Dial Transplant 2012;27:2418-25.

9 Johansen KL. Exercise in the end-stage renal disease population. $J$ Am Soc Nephrol 2007;18:1845-54.

10 Delgado C, Johansen KL. Barriers to exercise participation among dialysis patients. Nephrol Dial Transplant 2012;27:1152-7.

11 Jhamb M, McNulty ML, Ingalsbe G, et al. Knowledge, barriers and facilitators of exercise in dialysis patients: a qualitative study of patients, staff and nephrologists. BMC Nephrol 2016;17:1-14.

12 Mathiowetz V, Matuska KM, Murphy ME. Efficacy of an energy conservation course for persons with multiple sclerosis. Arch Phys Med Rehabil 2001;82:449-56.

13 Farragher JF. Developing "PEP": a personalized, web-supported energy conservation education program for people on chronic dialysis therapy with fatigue, 2018. Available: https://tspace.library. utoronto.ca/handle/1807/91865 [Accessed 23 Apr 2019].
14 Matuska K, Mathiowetz V, Finlayson M. Use and perceived effectiveness of energy conservation strategies for managing multiple sclerosis fatigue. Am J Occup Ther 2007;61:62-9.

15 Farragher JF, Jassal SV, McEwen S, et al. Development of an energy management education program ("the PEP program") for adults with end-stage renal disease. $\mathrm{Br} J$ Occup Ther 2020;83:397-404.

16 A proof-of-concept investigation of an energy management education program to improve fatigue and life participation in adults on chronic dialysis - Janine F, Farragher, Helene J. Polatajko, Sara McEwen, Sarbjit V. Jassal, 2020. Available: https://journals.sagepub. com/doi/full/ [Accessed 8 Oct 2020].

17 Baigent C, Herrington WG, Coresh J, et al. Challenges in conducting clinical trials in nephrology: conclusions from a kidney diseaseimproving global outcomes (KDIGO) controversies conference. Kidney Int 2017;92:297-305

18 Natale P, Gutman T, Howell M, et al. Recruitment and retention in clinical trials in chronic kidney disease: report from national workshops with patients, caregivers and health professionals. Nephrol Dial Transplant 2020;35:755-64.

19 Farragher JF, Thomas C, Ravani P, et al. Protocol for a pilot randomised controlled trial of an educational programme for adults on chronic haemodialysis with fatigue (Fatigue-HD). BMJ Open 2019;9:e030333.

20 Krupp LB, LaRocca NG, Muir-Nash J, et al. The fatigue severity scale. Application to patients with multiple sclerosis and systemic lupus erythematosus. Arch Neurol 1989;46:1121-3.

21 Cohen J. Statistical power analysis for the behavioral sciences. Academic Press, 2013.

22 Whitehead AL, Julious SA, Cooper CL, et al. Estimating the sample size for a pilot randomised trial to minimise the overall trial sample size for the external pilot and main trial for a continuous outcome variable. Stat Methods Med Res 2016;25:1057-73.

23 Arora P, Kausz AT, Obrador GT, et al. Hospital utilization among chronic dialysis patients. J Am Soc Nephrol 2000;11:740-6.

24 Molnar AO, Moist L, Klarenbach S, et al. Hospitalizations in dialysis patients in Canada: a national cohort study. Can J Kidney Health Dis 2018;5:205435811878037.

25 Farragher JF, Jassal SV, McEwen S, et al. Energy management education and occupation-related outcomes in adults with chronic diseases: a scoping review. Br J Occup Ther 2020;83:561-75.

26 Law M, Baptiste S, McColl M, et al. The Canadian occupational performance measure: an outcome measure for occupational therapy. Can J Occup Ther 1990;57:82-7.

27 Carswell A, McColl MA, Baptiste S, et al. The Canadian occupational performance measure: a research and clinical literature review. Can $J$ Occup Ther 2004;71:210-22.

28 Wood-Dauphinee SL, Opzoomer MA, Williams Jl, et al. Assessment of global function: the reintegration to normal living index. Arch Phys Med Rehabil 1988;69:583-90.

29 Bourget N, Deblock-Bellamy A, Blanchette AK, et al. Use and psychometric properties of the reintegration to normal living index in rehabilitation: a systematic review. Ann Phys Rehabil Med 2018;61:262-9.

30 Learmonth YC, Dlugonski D, Pilutti LA, et al. Psychometric properties of the fatigue severity scale and the modified fatigue impact scale. $J$ Neurol Sci 2013;331:102-7.

31 Fisk JD, Ritvo PG, Ross L, et al. Measuring the functional impact of fatigue: initial validation of the fatigue impact scale. Clin Infect Dis 1994;18:S79-83.

32 Ju A, Teixeira-Pinto A, Tong A, et al. Validation of a core patientreported outcome measure for fatigue in patients receiving hemodialysis: the SONG-HD fatigue instrument. Clin J Am Soc Nephrol 2020;15:1614-21. 